\title{
Safe use of subdermal needles for intraoperative monitoring with MRI
}

\author{
Terrance M. Darcey, PhD,, ${ }^{1,2}$ Erik J. Kobylarz, MD, PhD, ${ }^{1}$ Michael A. Pearl, RT, ${ }^{3}$ \\ Patricia J. Krauss, MEd, CNIM, ${ }^{1}$ Stephanie A. Ferri, CNIM, ${ }^{1}$ David W. Roberts, MD, ${ }^{2}$ and \\ David F. Bauer, MD²
}

\begin{abstract}
${ }^{1}$ Department of Neurology, ${ }^{2}$ Section of Neurosurgery, ${ }^{3}$ Center for Surgical Innovation, Dartmouth-Hitchcock Medical Center, Lebanon, New Hampshire
\end{abstract}

OBJECTIVE The purpose of this study was to develop safe, site-specific procedures for placing and leaving subdermal needle leads for intraoperative monitoring (IOM) during intraoperative MRI procedures.

METHODS The authors tested a variety of standard subdermal needle electrodes designed and FDA-approved for IOM in the conventional operating room. Testing was used to determine the conditions necessary to avoid thermal injury and significant image artifacts with minimal disruption of IOM and MRI procedures. Phantom testing was performed with a fiber optic (lead) temperature monitoring system and was followed by testing of leads placed in a healthy volunteer. The volunteer testing used electrode placements typical of standard IOM cases, together with radiofrequency (RF) coil placement and imaging sequences routinely employed for these case types. Lead length was investigated to assess heating effects for electrodes placed within the RF coil.

RESULTS The authors found that conventional stainless steel (SS) and platinum/iridium (Pt/lr) subdermal needles can be used safely without significant heating when placed outside the RF coil, and this accounts for the majority or entirety of electrode placements. When placed within the RF coil, Pt/Ir leads produced minimal image artifacts, while SS leads produced potentially significant artifacts. In phantom testing, significant heating was demonstrated in both SS and Pt/ Ir leads placed within the RF coil, but only during high-resolution T2-weighted scanning. This problem was largely, but not completely, eliminated when leads were shortened to $25 \mathrm{~cm}$. Human testing was unremarkable except for nonpainful heating detected in a few electrodes during thin-slice $(1.5 \mathrm{~mm})$ FLAIR scanning. Transient irritation (skin reddening along the needle tract) was noted at 2 of the electrodes with detectable heating.

CONCLUSIONS The authors were satisfied with the safety of their site-specific procedures and have begun with offlabel use (following institutional review board approval and obtaining patient informed consent) of tested monitoring leads in cases that combine IOM and MRI. The authors recommend that all facilities perform their own site-specific testing of monitoring leads before proceeding with their routine use.

http://thejns.org/doi/abs/10.3171/2015.12.FOCUS15555

KEY WORDS intraoperative monitoring; intraoperative MRI; MRI safety testing; MRI thermal injury; MRI artifact

I NTRAOPERATIVE monitoring (IOM) has been used in the operating room (OR) for more than 2 decades and provides a means of neurophysiological evaluation in surgical patients under general or local anesthesia who have significant neurological risk. ${ }^{12}$ A wide variety of monitoring modalities are available to assist surgeons in managing surgical risk through ongoing electrophysiological testing and mapping procedures on the brain, cranial nerves, spi- nal cord, and peripheral nerves. In recent years, a number of facilities have introduced an intraoperative MRI capability, ${ }^{11}$ which raises issues for the safe and effective use of standard IOM leads during MRI. After our medical center recently obtained an intraoperative MRI scanner, we were motivated to develop procedures for doing IOM safely and efficiently in the MRI environment. IOM typically employs subdermal needle electrodes inserted at

ABBREVIATIONS COM = common; EEG = electroencephalography; EMG = electromyography; FLAIR = fluid attenuated inversion recovery; FO = fiber optic; FOV = field of view; IOM = intraoperative monitoring; MPRAGE = magnetization-prepared rapid gradient echo; OR = operating room; $\mathrm{Pt} / \mathrm{Ir}=\mathrm{platinum} / \mathrm{iridium}$; RF = radiofrequency; $\mathrm{SS}=$ stainless steel; SSEP = somatosensory evoked potential; tcMEP = transcranial motor evoked potential; TSE = turbo spin echo.

SUBMITTED October 30, 2015. ACCEPTED December 2, 2015.

INCLUDE WHEN CITING DOI: 10.3171/2015.12.FOCUS15555 
numerous locations on the body for recording and stimulation purposes, which raises significant image quality and safety considerations. Imaging artifacts can be caused by the material composition of the electrodes, leads, and securements. ${ }^{7}$ The primary safety risk is thermal injury due to electrode or lead heating in the MRI scanner, as a number of studies have reported thermal injury associated with MRI and other types of leads (i.e., electrocardiogram and pulse oximeter leads). ${ }^{3,5,6}$ Unfortunately, in practice, MRI heating effects are relatively unpredictable and do not transfer well across different facilities and operating conditions..$^{1,13,14}$

At the time of this writing, there are no available FDAapproved, MR-safe, or MR-conditional subdermal needle leads that are also appropriate for general IOM use. The "MR-safe" label rarely appears in MRI safety information, while "MR-conditional" is more common, with labeled items having been tested and approved for specific scanning equipment and parameters..$^{15}$ Given the complexity and idiosyncrasies of each MRI facility and its equipment, we believe that site-specific safety testing should be performed even when MR-conditional leads become available and when changes in MRI equipment, scanning sequences, or leads are contemplated.

Our standard operating procedure for performing IOM with our MRI scanner in its 1st year of operation was to 1) place the leads after inducing anesthesia and performing any preoperative MRI studies, 2) remove and account for our monitoring leads under the sterile drapes prior to intraoperative MRI, and, if necessary, 3) replace our monitoring leads without compromising the sterile field to resume monitoring. Most other centers in the US and abroad reportedly follow this same (removal/replacement) approach for intraoperative MRI when performing IOM. However, removal/replacement significantly extends the turnaround time for intraoperative MRI, and some risks are involved in accessing leads that are under surgical drapes, especially those near or within the sterile field. Many of the lead locations may not be visible once under the sterile drapes, causing lead removal/replacement to be done by feel, which increases the risk of needle sticks, inadvertent pullout of intravenous catheters or other anesthesia lines, contamination of the sterile field, and potential modification of the patient's original positioning and padding.

Our goal was to develop safe, site-specific procedures for placing and leaving subdermal needle leads for intraoperative MRI studies. It should be noted that our IOM equipment (amplifiers, stimulators, cables, computerized monitoring system) is not MR compatible, and we do not contemplate the need to monitor during intraoperative MRI.

\section{Methods \\ Leads and Imaging Procedures}

Our testing focused on 1) safety in terms of avoiding possible thermal injury due to lead heating, and 2) the assessment of image quality to avoid image artifacts arising from lead materials affecting the MRI field of view (FOV). Various candidate platinum/iridium (Pt/Ir) and stainless steel (SS) subdermal needle leads were used for phantom testing (Table 1). All subdermal needle leads were designed and FDA approved for IOM use in the regular OR, had standard DIN plugs for connection to IOM equipment, were packaged sterile, and were available for purchase. Additional testing was performed to assess the heating characteristics of SS surgical staples (Visistat 35 W, Teleflex Medical Inc.) that are typically used to secure needles on the scalp within the hairline.

A total of 12 separate MRI sessions were completed over the course of 2 months, including 6 phantom and 6 healthy volunteer tests. Scanning was performed in our intraoperative MRI scanner (IMRIS Inc./Skyra 3T, Siemens Medical Solutions, Inc.) using the same radiofrequency (RF) coil arrangement (2 intraoperative, 4-channel, receive-only phased-array flex coils, 1 upper and 1 lower for a total of 8 channels) and imaging sequences employed for intraoperative MRI. This included T1-weighted magnetization-prepared rapid gradient echo (MPRAGE), T2weighted turbo spin echo (TSE), and T2-weighted fluid attenuated inversion recovery (FLAIR) imaging, which were run repeatedly while changing the lead orientation and position within and with respect to the RF coil. Both thin- and thick-slice (1.5 and $3 \mathrm{~mm}$, respectively) FLAIR scans were investigated because electrode heating was noted during thin-slice FLAIR imaging when leads were placed within the RF coil (Table 2).

\section{Phantom Lead Testing}

Our initial tests evaluated subdermal needle electrodes placed in a phantom (honeydew melon approximating the size of a human head). The electrodes were inserted at a relatively shallow angle under the melon skin to mimic the subdermal placement typically used for electroencephalography (EEG), electromyography (EMG), and stimulation leads and at various orientations within, at the edges of, and well outside the RF coil. Initial scanning was done with the leads (4 at a time given the limitation of 4 temperature measurement channels) at their manufactured length, using various schemes for configuring and routing the electrode tails out of the magnet. Lead length was investigated in some detail to assess the heating effects of MRI on leads placed within the RF coil.

TABLE 1. Subdermal needle leads tested

\begin{tabular}{lcccl}
\hline \multicolumn{1}{c}{ Description } & Material & Dimensions $(\mathrm{mm})$ & Tail $(\mathrm{m})$ & \multicolumn{1}{c}{ Manufacturer } \\
\hline Straight needle & Pt/lr & $0.4 \times 12$ & 1.5 & Technomed \\
\hline Straight needle & SS & $0.4 \times 13$ & 2.5 & Rhythmlink International LLC \\
\hline Straight needle pair, twisted & SS & $0.4 \times 13$ & 2.0 & Medtronic plc \\
\hline Straight needle pair, parallel & SS & $0.4 \times 13$ & 1.5 & Rhythmlink International LLC \\
\hline Corkscrew needle & SS & 0.4 spiral & 1.2 & Medtronic plc \\
\hline
\end{tabular}


TABLE 2. Parameters of MRI sequences

\begin{tabular}{|c|c|c|c|c|}
\hline Scan Parameter & T1 MPRAGE & T2 TSE & T2 FLAIR Thin & T2 FLAIR Thick \\
\hline $\mathrm{TR}$ (msec) & 1900 & 4100 & 12,200 & 8000 \\
\hline TE (msec) & 2.55 & 104 & 84 & 81 \\
\hline $\mathrm{TI}$ (msec) & 900 & NA & 2772 & 2372 \\
\hline FOV (mm) & 240 & 240 & 240 & 240 \\
\hline Matrix & $512 \times 512 \times 120$ & $320 \times 320 \times 90$ & $320 \times 320 \times 90$ & $320 \times 320 \times 45$ \\
\hline Resolution (mm) & 0.47 & 0.75 & 0.75 & 0.75 \\
\hline Slice $(\mathrm{mm})$ & 1.5 & 1.5 & 1.5 & 3.0 \\
\hline $\mathrm{FA}\left({ }^{\circ}\right)$ & 9 & 140 & 133 & 133 \\
\hline SAR (W/kg) & 0.008 & 0.09 & 0.15 & 0.09 \\
\hline Average RF power (W) & 4.9 & 55.1 & 56.8 & 43.2 \\
\hline Duration (sec) & 375 & 432 & 368 & 242 \\
\hline
\end{tabular}

$\mathrm{FA}=$ flip angle; $\mathrm{NA}=$ not available; $\mathrm{SAR}=$ specific absorption rate; $\mathrm{TE}=$ echo time; $\mathrm{TI}=$ inversion time; $\mathrm{TR}=$ repetition time.

During phantom scanning, ongoing lead temperature measurement was performed using an MR-compatible, 4-channel, fiber optic (FO) temperature measurement system (Reflex, Neoptix Corp.). The FO sensors, which extended $5 \mathrm{~m}$ from the recorder (not MR compatible), recorded the temperature every second with a resolution of $\pm 0.1^{\circ} \mathrm{C}$. Lead temperatures were measured with the FO sensors placed 1) under the melon skin parallel to the leads and separated by approximately $1 \mathrm{~mm}$, and 2) in almost direct contact with the needle lead at its entry point through the melon skin. The second method was quickly adopted for most of the phantom testing, as it gave the most consistent and direct temperature measurements (Fig. 1).

\section{Lead Testing in a Healthy Volunteer}

A healthy volunteer (T.M.D.) was used to assess heating effects by placing leads to simulate typical IOM cases, including brain and upper and lower spine surgeries. Testing on a single individual was considered appropriate for the immediate aims of our site-specific safety testing, as opposed to a systematic research study intended to generalize findings to other facilities, which would have also required institutional review board approval. To assess lead temperature, we relied on the volunteer's report of sensory changes during MRI and inspected the electrode sites after scanning. This was considered the most sensitive measurement technique under the circumstances. Using the FO temperature sensors on a healthy volunteer would be problematic from a practical standpoint, since the sensors would be difficult to sterilize and safely place in near contact with inserted needles without risking infection. How this might be facilitated in the future is considered in the Discussion below.

As with the phantom testing, multiple subdermal lead types from 3 different vendors were tested in the MRI scanner. Securement and placement techniques were also evaluated to identify potential safety or image quality issues. Except for the Pt/Ir leads, which were sutured in place with silk ties, securement and placement techniques were the same as those used in the regular OR. Additionally, strain-relieving loops were avoided. Leads outside the hairline were secured using surgical adhesive tape (Blenderm,
3M Healthcare) combined with liquid adhesive (Mastisol, Ferndale Labs). Leads within the hairline were similarly secured given that surgical staples and sutures could not be used without undue focal discomfort and consequent interference with sensory detection of electrode heating. In 1 case, a surgical staple was placed on the scalp.

\section{Results}

\section{Phantom Lead Testing}

As expected, the Pt/Ir leads created minimal imaging
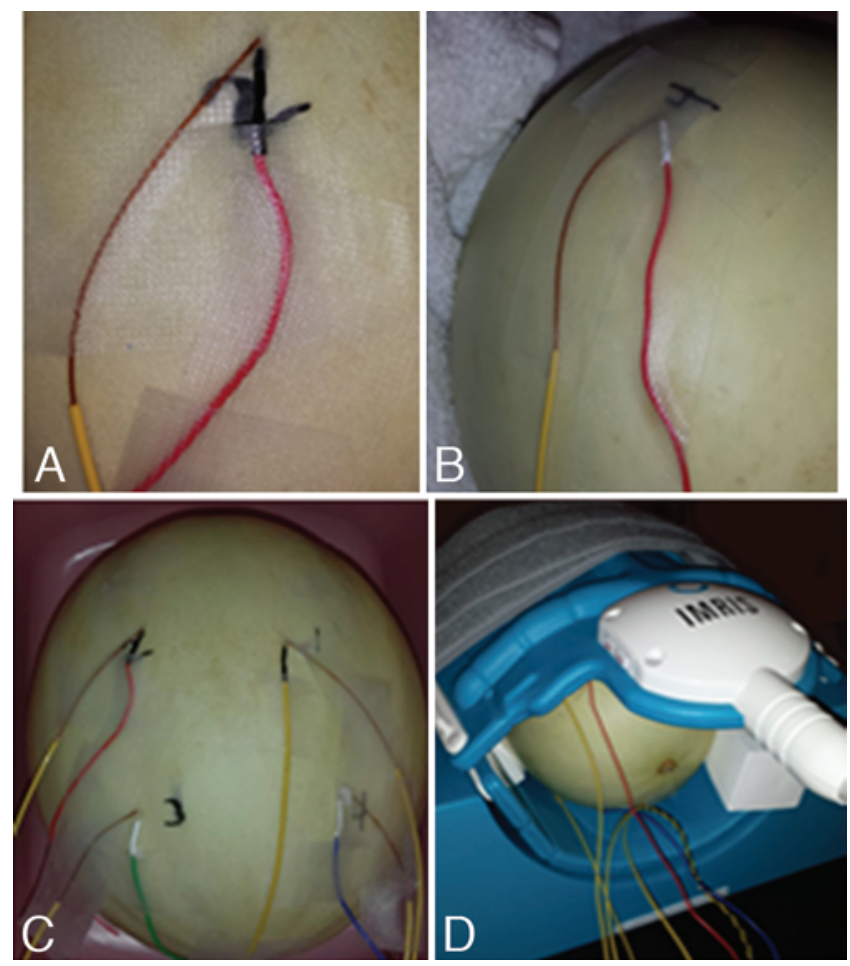

FIG. 1. Images of phantom testing with a melon, $2 \mathrm{Pt} / \mathrm{lr}$ and $2 \mathrm{SS}$ subdermal leads, and FO temperature sensors in place, illustrating placement of the FO sensors proximal to and in contact with the needle at its entry point $(\mathbf{A}-\mathbf{C})$ and how the phantom was situated in the RF coil for MRI (D). 
artifacts, while the SS leads and surgical staples created significant localized artifacts up to $2 \mathrm{~cm}$ in diameter (Fig. 2 ). In the first 4 sessions, no significant temperature changes $\left(>4^{\circ} \mathrm{C}\right)$ were measured, and no clear inductive RF heating was observed with intentional looping and crossing of leads. In the fifth session, significant temperature changes were noted during T2-weighted scanning for 1 of the Pt/ Ir and 1 of the SS electrodes, with maximum transient temperature changes of approximately $15^{\circ} \mathrm{C}$ in thin-slice FLAIR and $6^{\circ} \mathrm{C}$ in TSE sequences. This prompted immediate experimentation with lead lengths (through trimming of the electrode tails) to see if temperature increases could be reduced or eliminated. This line of investigation was prompted by reports ${ }^{2,5}$ of RF antenna-effect heating at particular lead lengths related to the MRI resonant wavelength. In fact, during T2-weighted imaging, heating was greatly diminished when leads were shortened to $40 \mathrm{~cm}$ and substantially eliminated when leads were shortened to $25 \mathrm{~cm}$ (Fig. 3).

Our summary assessment at this point was as follows: 1) leads of any type were safe in terms of heating when placed well outside the RF coil or subjected to T1 imaging only, 2) we needed to do further testing on the effects of lead length on heating during T2-weighted imaging, and 3 ) we would probably need to restrict lead length to prevent the heating of leads placed within the RF coil when performing thin-slice FLAIR and TSE scans. We also examined available information on lead length and focused on the 3 types of scalp leads with MR conditional FDA labeling (Table 3). These leads all have short tail lengths, ranging from 13 to $25 \mathrm{~cm}$, and were packaged with removable MR-incompatible extensions of 200 to $250 \mathrm{~cm}$. Based on this information, we decided to adopt $25 \mathrm{~cm}$ as the length we would pursue for leads placed within the RF
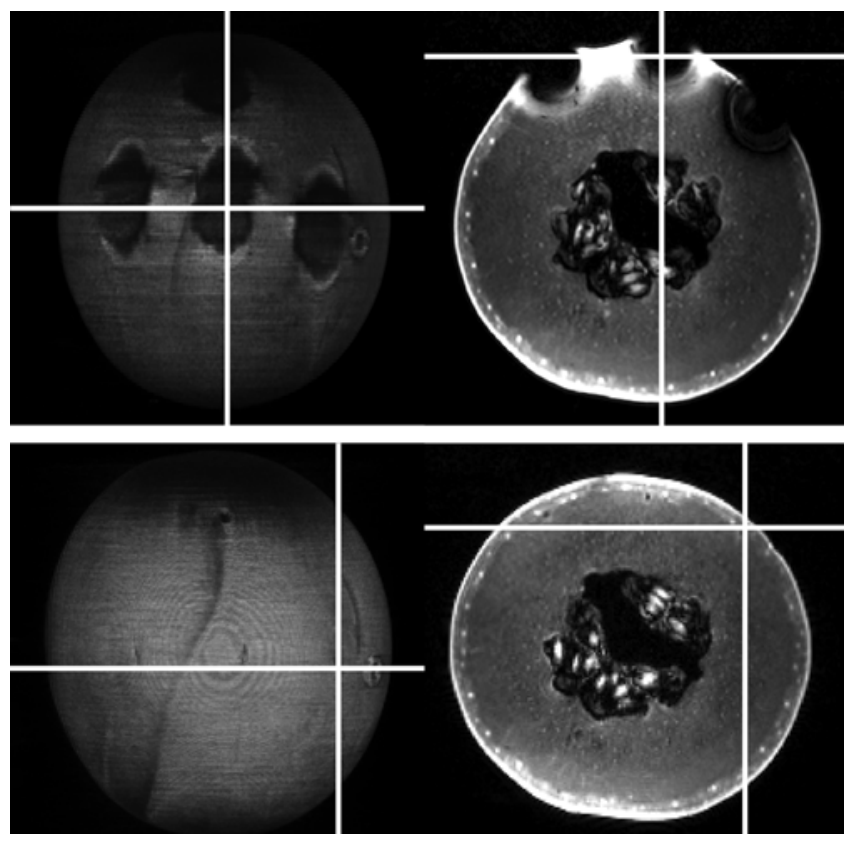

FIG. 2. MRI studies of the melon phantom with SS (upper) and Pt/Ir (lower) subdermal electrodes and FO temperature sensors in place. Left panels show surface renderings, while the right panels show individual slices from high-resolution T1 MPRAGE images.

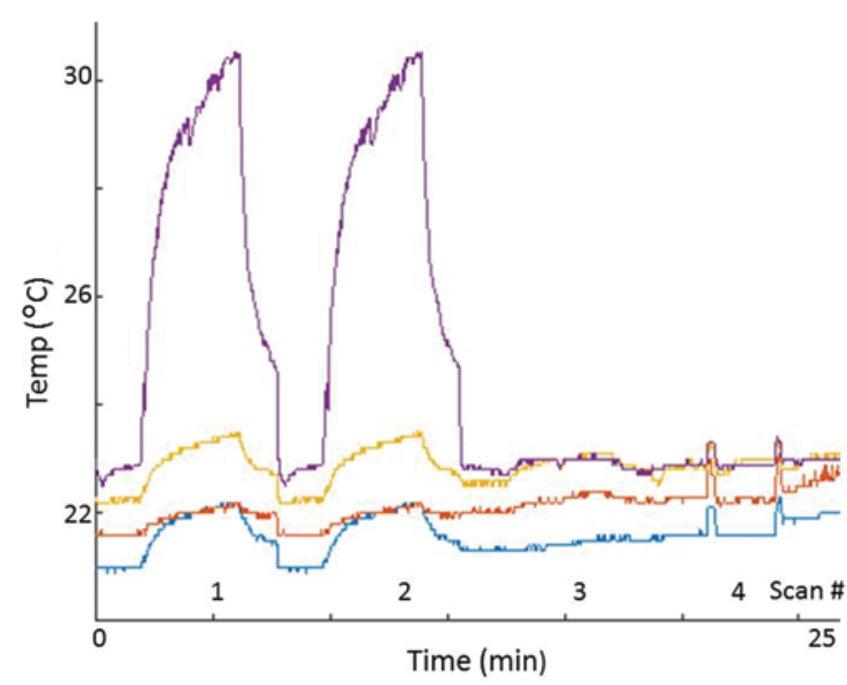

FIG. 3. FO temperature traces for thin-slice FLAIR scans of the melon phantom with $1 \mathrm{Pt} / / \mathrm{r}$ (purple trace) and $3 \mathrm{SS}$ (blue, red, yellow traces) subdermal leads in place, all within the RF coil. Temperature data are plotted for 4 sequential scans of 6 minutes, 8 seconds' duration each, with incremental shortening of lead tails. Scan $1=$ standard length; Scan $2=150 \mathrm{~cm}$; Scan $3=40 \mathrm{~cm}$; Scan $4=25 \mathrm{~cm}$. Heating of up to $15^{\circ} \mathrm{C}$ was noted in the $\mathrm{Pt} / \mathrm{Ir}$ electrode with lead extensions in place.

coil, with the plan of using similar removable extensions to connect to IOM equipment, which would be disconnected and removed for intraoperative MRI. We should note that we had previously obtained evaluation samples of the above electrodes and concluded that none was appropriate for general IOM use. For subdermal needles with a reduced lead length of $25 \mathrm{~cm}$, no additional significant temperature increases were measured in our phantom studies. At this point, we decided that it would be safe to test subdermal Pt/Ir and SS leads in a healthy volunteer.

\section{Lead Testing in a Healthy Volunteer}

The first MRI test was performed with leads placed on the foot and lower leg of the healthy volunteer, with his foot inside the RF coil. Full-length leads (1.5-2.5 m) were used, and no heating or skin irritation and/or injury was noted for any lead during MPRAGE, TSE, and thinslice FLAIR imaging. Next, we performed extensive lead placements for 3 common types of surgery, followed by MRI while the leads were left in place. Based on results of the phantom tests, leads within the RF coil were shortened to $25 \mathrm{~cm}$ after MPRAGE imaging and prior to TSE or FLAIR imaging. As expected, MPRAGE imaging resulted in no significant heating of leads of any type or at any length when placed inside or outside the RF coil, and no heating was detected with any of the imaging modalities when leads were placed outside the RF coil. It should be emphasized that this accounts for the overwhelming majority of leads typically placed for IOM. And, as expected, imaging artifacts were insignificant for $\mathrm{Pt} / \mathrm{Ir}$ leads placed within the RF coil but were potentially significant $(1-2 \mathrm{~cm}$ diameter) for SS leads and surgical staples within the RF coil; therefore, one must choose between these electrode types on a case-by-case basis when placing leads within the RF coil. As detailed below, no differences were seen 
TABLE 3. Currently available, FDA-approved, MR-conditional scalp EEG leads

\begin{tabular}{llccc}
\hline \multicolumn{1}{c}{ Electrode/Description } & \multicolumn{1}{c}{ Material } & $\begin{array}{c}\text { Tail Length } \\
(\mathrm{cm})\end{array}$ & $\begin{array}{c}\text { Lead Extensions } \\
(\mathrm{cm})\end{array}$ & Manufacturer \\
\hline Press-on (3-prong subdermal w/ applicator) & Nickel titanium & 25 & 250 & Rhythmlink International LLC \\
\hline MR-conditional cup (surface glue on, electrolyte gel) & Plastic, $\mathrm{Ag} / \mathrm{AgCl}$ coating & 24 & $150-250$ & Rhythmlink International LLC \\
\hline SWE (subdermal wire w/ needle applicator) & Silver wire, $\mathrm{AgCl}$ coating & $13-25$ & $150-250$ & Ives EEG Solutions Inc. \\
\hline
\end{tabular}

between the Pt/Ir and SS lead types in terms of their heating characteristics.

We first tested the lead setup for lumbar-sacral spine surgery requiring transcranial motor evoked potential (tcMEP), somatosensory evoked potential (SSEP), and EMG monitoring (Fig. 4). Full-length (2 m), twisted-pair SS leads were used exclusively, with EMG lead pairs placed in the bilateral upper (first dorsal interosseous) and lower (adductor longus, vastus lateralis, tibialis anterior, gastrocnemius, abductor hallucis brevis) extremity muscles; EEG and/or SSEP leads placed at scalp locations $\mathrm{FPz}, \mathrm{CPz}, \mathrm{CP} 3$, and CP4; SSEP and train-of-four stimulating lead pairs placed over the bilateral ulnar and posterior tibial nerves; tcMEP stimulating leads placed at scalp locations $\mathrm{C} 1$ and $\mathrm{C} 2$; and common (COM) electrodes placed on 1 upper and 1 lower extremity. Anal sphincter leads typically used in such cases were not applied, as these leads would be difficult to tolerate for a healthy awake volunteer. Since anal sphincter leads would often be within the RF coil in this type of case, short-tailed leads would probably need to be used if T2weighted scanning was performed. The healthy volunteer was prone for MRI, just as he would be positioned for this type of surgery, and no heating effects or image distortions were noted for any type of MRI. This was not unexpected, as no leads were placed within the RF coil.

The lead setup for cervical spine surgery was similar to that for lumbar-sacral spine surgery, except that we used a different selection of EMG leads and a different placement scheme for the tcMEP scalp leads. EMG leads were placed in the bilateral upper (trapezius, deltoid, biceps, extensor carpi radialis, first dorsal interosseous) and lower (adductor longus, tibialis anterior, abductor hallucis brevis) extremity muscles, and tcMEP stimulating leads were placed at scalp locations $\mathrm{C} 3$ and $\mathrm{C} 4$ rather than $\mathrm{C} 1$

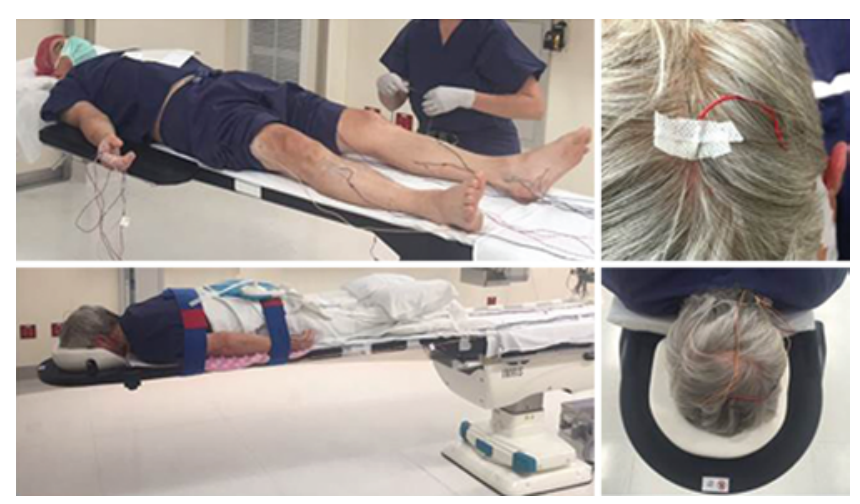

FIG. 4. Images of healthy volunteer setup, with lead placements simulating a lumbar-sacral spine surgery with intraoperative MRI. In this case, all leads were SS with full-length tails, since no leads were placed within the RF coil. and $\mathrm{C} 2$. The remaining stimulating scalp EEG and COM leads were placed in the same locations as for lumbarsacral spine surgery. Full-length SS twisted-pair leads were used exclusively, as none would be within the RF coil. The volunteer was prone for MRI, just as he would be positioned for this type of surgery, and no heating effects or image distortions were noted for any type of MRI. As before, these results were not surprising, since no leads were placed within the RF coil.

The third lead setup was for right-sided brain tumor surgery. EMG lead pairs were placed in the contralateral face (masseter, orbicularis oris), upper extremity (trapezius, deltoid, biceps, extensor carpi radialis, first dorsal interosseous, adductor digiti minimi, abductor pollicis brevis), and lower extremity (adductor longus, vastus lateralis, tibialis anterior, gastrocnemius abductor hallucis brevis) muscles. SSEP and train-of-four stimulating leads were applied to the contralateral wrist and ankle, a COM lead was placed on the shoulder, and scalp EEG leads were placed in areas $\mathrm{FPz}, \mathrm{CPz}, \mathrm{CP} 3$, and $\mathrm{CP} 4$. The tongue leads typically used in such cases were not applied, as these leads would have been difficult to tolerate for a healthy awake volunteer. Since tongue leads would be within the RF coil in this instance, short-tailed leads would probably need to be used if T2-weighted scanning was performed. Lead removal/replacement probably would be done pending appropriate safety testing. If T2-weighted scanning was performed, this uncertainty with regard to safety would generally apply to untested lead placements, including the tongue, soft palate, eye muscles, and anal sphincter. MPRAGE scanning was performed in 3 head positions (right side up, supine, left side up), and no heating was noted in any lead. The head and face leads were trimmed to roughly $25 \mathrm{~cm}( \pm 2 \mathrm{~cm})$ prior to T2 FLAIR thin-slice scanning. Transient, nonpainful heating was clearly noted on the CP3 scalp electrode in the right-side-up position, on the $\mathrm{Cpz}$ electrode in the supine position, and on the CP3 and FPz electrodes in the left-side-up position. The heating was a pulsatile, pinprick sensation at the electrode site, correlated to the pulse sequence, with rapid extinction of the sensation between pulses. Inspection of the electrode sites after scanning revealed visible skin irritation at the FPz electrode site only, characterized as redness along the needle track (Fig. 5). This skin irritation was not persistent, did not require treatment, and was no longer visible a few days after the procedure. However, this irritation established that electrode heating was still possible with shortened leads and suggested the need for further tests focusing on scalp leads placed within the RF coil when T2 or other high-energy sequences were required.

The next scanning session used a larger array of scalp leads (12 positions including FPz, Fz, F3, Cz, C3, P3, Pz, 

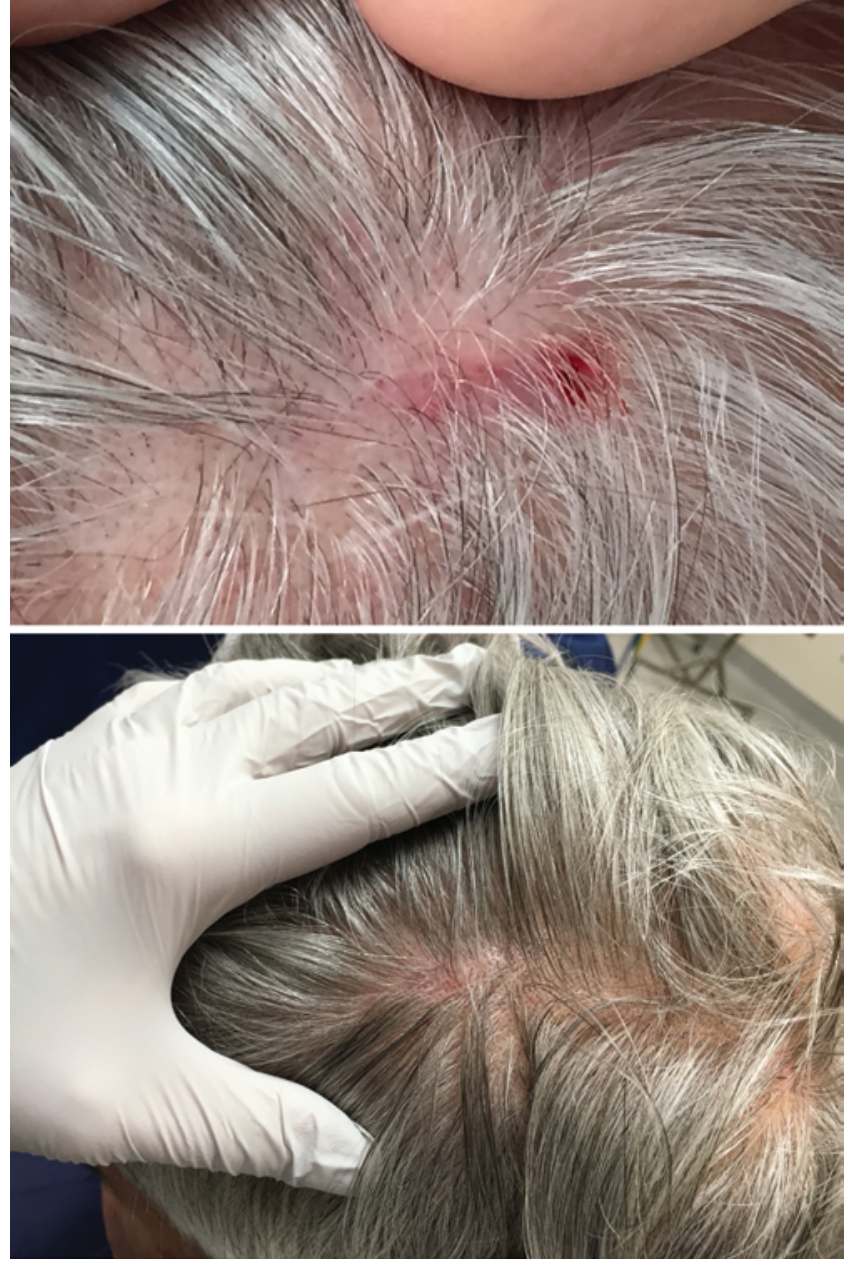

FIG. 5. Skin reddening noted at 2 electrode sites following thin-slice FLAIR scanning in a healthy volunteer. Nonpainful heating was reported for these and other scalp leads placed within the RF coil. Upper: Vicinity of the FPz electrode in the scalp in a setup simulating right-sided brain tumor surgery, with head leads in place and with the tails trimmed from standard length to $25 \mathrm{~cm}$. The dark red blotch is residual blood near the needle entry point. Lower: Scalp in the vicinity of the C3 electrode in a final test using 12 scalp leads with $25-\mathrm{cm}$ tails terminated with DIN plugs.

A1, F4, C4, A2, P4) that were pretrimmed to precisely 25 $\mathrm{cm}$ and all placed within the RF coil. In this case, we focused on thin-slice and thick-slice $(1.5$ and $3 \mathrm{~mm}$, respectively) FLAIR sequences obtained for the 3 head positions (supine, right side up, left side up). No detectable heating or skin irritation was noted for any of the sequences or head positions.

After the aforementioned tests, we believed there was sufficient evidence to conclude that for FLAIR scanning, the tested leads would be safe if restricted to $25 \mathrm{~cm}$ when placed within the RF coil, although it remained possible that some heating and skin irritation could occur even with these precautions. After testing, we obtained institutional review board approval for off-label use of the tested leads at our facility with informed consent by the patient and have since used them without incident (no skin irritation evident at any electrode site) in 5 patients, including 2 who underwent cervical spine surgery and 3 who had surgery for brain tumor. For these patients, electrodes within the
$\mathrm{RF}$ coil were either trimmed or removed/replaced when T2-weighted scanning was performed.

We next secured factory-made Pt/Ir and SS leads with $25-\mathrm{cm}$ tails. This electrode tail length was considered a reasonable precaution considering 1) the mixed results of the prior 2 scanning sessions using scalp leads inside the RF coil (1 session demonstrating heating and the other not), and 2) the fact that these leads had DIN connectors as opposed to cut ends. For this test, a mix of Pt/Ir and SS leads were placed on the scalp (Fz, Cz, F4, C4, P4 for Pt/Ir; F3, C3, P3, Pz for SS), and 3 head positions were tested, including supine, right side up, and left side up, with a focus on FLAIR scanning. As before, no heating effects were noted for MPRAGE or TSE scanning. Transient, nonpainful heating was clearly noted at the F4 and P3 scalp electrodes in all 3 positions with thin-slice FLAIR scanning. The heat was a pulsatile, pinprick sensation that correlated to the pulse sequence, with rapid extinction of the sensation between pulses. This sensation was very similar in character to, but less intense than, that in the prior session in which there was detectable electrode heating. Trace sensations were still detectable for thick-slice FLAIR scanning. Inspection of the electrode sites after scanning revealed visible skin irritation at the $\mathrm{C} 3$ electrode site only, characterized as redness along the needle track. This skin irritation was not persistent, did not require treatment, and was no longer visible a few days after testing.

\section{Discussion}

The purpose of our site-specific safety testing was to establish in-house guidelines for the safe use of subdermal needle electrodes for IOM during intraoperative MRI. Establishing these guidelines was meant to obviate the removal and replacement of leads when monitoring is interrupted for an MR image. Before undertaking this study, we were encouraged by reports from other groups who described the successful use of subdermal needles in their facilities. ${ }^{4,9}$ We emphasize that our results do not generalize to other facilities, electrode types, or scanning sequences. We fully expect to do similar, repeated testing for any significant changes at our site, such as MRI hardware, scanning sequences, or lead types. We recommend similar testing at other facilities prior to the adoption of techniques for routine patient use.

The importance of keeping IOM in place during intraoperative MRI should be emphasized. Removal and replacement of needle electrodes from underneath sterile drapes poses a real risk of sterile field contamination. Electrode removal takes extra time given the difficulty in working underneath the sterile drape, and electrode replacement can disrupt careful patient positioning, leading to potential pressure sores from misplaced bolsters or padding. Because of this difficulty, a surgeon may continue tumor resection without IOM, which could increase operative morbidity.

A few lead types (conductive plastic cup, press-on, and silver hookwire electrodes, see below) have been developed for scalp EEG recording and are FDA labeled as MR conditional, but these leads are not appropriate or adaptable for general IOM use, which requires leads appropriate 
for EEG, EMG, and electrical stimulation at many body locations. Subdermal needle electrodes have proven most suitable for these combined purposes. A few institutions have reported safe and effective off-label use of relatively standard, FDA-approved subdermal needle types that are left in place for MRI at their facilities. ${ }^{4,9}$ The problem to date has been that those are site- and equipment-specific findings that do not generalize to other facilities. We adopted a strategy of performing our own site-specific testing of subdermal needle electrodes that were FDA approved for IOM use in the conventional OR, with a view toward their potential off-label use in the MRI scanner. Pending an MR-conditional listing by the FDA, informed consent will be used to apprise patients of the off-label use of unapproved leads that have been tested locally to assure ourselves and the institutional review board of the nonsignificant risks to the patient.

In a review of the literature, we found a clear lack of consensus and empirical evidence on the physics and specific mechanism of thermal injuries in the MRI scanner. ${ }^{5}$ This reflects the fact that the MRI system is a complex electromagnetic environment into which the patient is placed with multiple leads and subcutaneous lines, forming a complex network of possible conducting paths internal and external to the patient. The standard explanation for heating in the MRI scanner is the induction of high eddy or lead currents by changing RF magnetic fields according to Faraday's law. ${ }^{10,14}$ Another, less commonly cited, explanation is the induction of resonant effects in tissue or leads by changing electric fields, typically referred to as "antenna effects.", tions of these effects under test conditions, but the specific configurations under which heating occurs have not risen to the point of predictability based on theory, making empirical testing a practical necessity. As such, all MRI equipment, scanning sequences, leads, and placement procedures should be tested prior to routine use and then reevaluated if any significant changes are made.

We acknowledge that there are limitations associated with phantom testing in a melon and the use of only $1 \mathrm{hu}-$ man volunteer. There are potential conductive differences between a melon and live human tissue with regard to heating. Live human tissue also exhibits potential protective mechanisms against overheating, such as sweating, that could help mitigate thermal injury. We believe our study was thorough and robust as regards the extent of testing in our melon and our human volunteer; however, we believe that further testing in other human volunteers could help validate our results and provide potential safety data prior to widespread patient use.

Our testing established that detectable and arguably significant electrode heating was possible in a narrow set of circumstances, even with the precaution of using shortened leads within the RF coil. This was seen with high-energy sequences such as thin-slice FLAIR. While we believe that the potential injuries associated with thinslice FLAIR would probably be tolerable to patients if such scans were critical to intraoperative decision making, we plan to avoid using these sequences because of the potential risk to our patients. Our findings suggest that lower-energy scans, such as thick-slice FLAIR, should be considered as an alternative to thin-slice FLAIR when possible. Our findings also reinforce the need to maintain close scrutiny of electrode sites within the RF coil when $\mathrm{T} 2$ or other high-energy sequences are used.

Whether the heating effects noted herein rise to the level of a significant tissue injury is a matter for discussion and clinical judgment; these effects should be considered in relation to the conventional use of subdermal leads and securement staples, which produce minor puncture wounds and skin irritation that may be visible for a few days after surgery. In the few instances in which heating was detected in our testing, our volunteer reported that the sensation was not painful, and visible skin irritation was rarely produced. It is pertinent to note that investigations of temperature thresholds for dermal injury have shown that nonpainful heating of even extended duration does not produce significant skin injury. ${ }^{6,8,16}$ While the threshold between an acceptable "minor" injury compared with an unacceptable "major" injury may vary from person to person, we believe that limiting imaging to lower-energy MR scans will keep potential thermal injuries at a level similar to the puncture wounds and skin irritation we typically encounter from traditional electrode placement.

A key breakthrough would be the development of a principled model that could predict and mitigate heating effects in the MRI scanner. This would help us and others to overcome the need for empirical testing and to devise more general and site-specific safety procedures. Simple, theoretical heating mechanisms such as Faraday induction and resonant antenna effects are hard to apply in practice. Improved measurement technologies capable of sensing lead temperature or induced currents would also be helpful, and there are a number of feasible options. One possibility is the development of a lead with a port into which an FO temperature probe could be inserted to make thermal contact with the lead without contaminating the subdermal needle. Another possibility is the use of infrared imaging technology that would allow visualization and measurement of the temperature distribution on the scalp or body surface, including the electrode sites. A third possibility is the use of MR-compatible, high-frequency electrical measurements (voltage or currents carried by the lead wires), which might be obtainable from unterminated DIN plugs on each lead. This latter possibility would perhaps allow detection of resonant or other high-current conditions and facilitate the introduction and testing of countermeasures, such as the use of additional components aimed at dampening resonant oscillations or dangerous induced currents.

\section{Conclusions}

First, conventional SS subdermal IOM needles with standard-length $(1.5-2.5 \mathrm{~m})$ tails can be safely used outside the RF coil without significant heating in the tested scan types (MPRAGE, TSE, and FLAIR). In most IOM and MRI cases, this use of IOM needles outside the RF coil will account for the majority or entirety of electrode placements. Second, in phantom testing, significant heating of some electrodes was demonstrated for standardlength (1.5-2.5 m) SS and Pt/Ir leads placed within the RF coil, but only during T2-weighted scanning (FLAIR > 
TSE), with no heating noted during T1-weighted scanning. $\mathrm{SS}$ and $\mathrm{Pt} / \mathrm{Ir}$ electrodes appeared equally likely to exhibit heating, and no clear pattern of which electrodes would heat was evident. The maximum recorded temperature increase in phantom testing was $15^{\circ} \mathrm{C}$ in a $\mathrm{Pt} / \mathrm{Ir}$ electrode. In addition, phantom testing of leads within the RF coil during T2-weighted scanning revealed the elimination of significant heating when leads were shortened to $25 \mathrm{~cm}$. Thus, we decided to use short-tailed leads for placements within the RF coil when T2-weighted scanning was required. Third, imaging artifacts were minimal for Pt/Ir electrodes but were potentially significant $(1-2 \mathrm{~cm}$ diameter) for SS electrodes within the RF coil and image FOV. Depending on the clinical circumstances, a mix of $\mathrm{Pt} / \mathrm{Ir}$ and SS leads could be used, e.g., placement of Pt/Ir leads over the left hemisphere and SS leads over the right hemisphere in a patient with a left-sided tumor. On a practical note, $\mathrm{Pt} / \mathrm{Ir}$ leads cost approximately 5 times as much as SS leads. Fourth, human testing was unremarkable except for nonpainful heating that was detected during thin-slice FLAIR scanning while using short-tailed $25-\mathrm{cm}$ leads within the RF coil. This heating occurred sporadically at a small number (2-3) of $\mathrm{Pt} / \mathrm{Ir}$ or SS electrodes in 2 of 3 sessions. Transient irritation (visible skin reddening along the needle tract) was noted at 2 of the electrodes with detectable heating. Heating became barely detectable when thick-slice FLAIR scanning was performed.

\section{Acknowledgments}

We thank Technomed for the evaluation samples of $\mathrm{Pt} / \mathrm{Ir}$ and SS needle electrodes.

\section{References}

1. Baker KB, Tkach JA, Nyenhuis JA, Phillips M, Shellock FG, Gonzalez-Martinez J, et al: Evaluation of specific absorption rate as a dosimeter of MRI-related implant heating. J Magn Reson Imaging 20:315-320, 2004

2. Balasubramanian M, Wells WM, Ives JR, Britz P, Lodenkemper T, Sundaram P, et al: RF heating of gold cup and conductive plastic EEG electrodes during simultaneous MRI and EEG. Presented at the Joint Annual Meeting ISMRMESMRMB, Milan, Italy, 2014 (Poster \#4876). (http://www. ismrm.org/14/program_files/EP18.htm) [Accessed January $11,2016]$

3. Bottomley PA: Turning up the heat on MRI. J Am Coll Radiol 5:853-855, 2008

4. D'Andrea G, Angelini A, Foresti C, Familiari P, Caroli E, Frati A: Platinum-iridium subdermal magnetic resonance imaging-compatible needle electrodes are suitable for intraoperative neurophysiological monitoring during image-guided surgery with high-field intraoperative magnetic resonance imaging: an experimental study. Neurosurgery 10 (Suppl 3):387-392, 2014
5. Dempsey MF, Condon B: Thermal injuries associated with MRI. Clin Radiol 56:457-465, 2001

6. Dewhirst MW, Viglianti BL, Lora-Michiels M, Hanson M, Hoopes PJ: Basic principles of thermal dosimetry and thermal thresholds for tissue damage from hyperthermia. Int $\mathbf{J}$ Hyperthermia 19:267-294, 2003

7. Gill A, Shellock FG: Assessment of MRI issues at 3-Tesla for metallic surgical implants: findings applied to 61 additional skin closure staples and vessel ligation clips. J Cardiovasc Magn Reson 14:3, 2012

8. Greenhalgh DG, Lawless MB, Chew BB, Crone WA, Fein ME, Palmieri TL: Temperature threshold for burn injury: an oximeter safety study. J Burn Care Rehabil 25:411-415, 2004

9. Hatiboglu MA, Weinberg JS, Suki D, Tummala S, Rao G, Sawaya R, et al: Utilization of intraoperative motor mapping in glioma surgery with high-field intraoperative magnetic resonance imaging. Stereotact Funct Neurosurg 88:345352,2010

10. Kanal E: An overview of electromagnetic safety considerations associated with magnetic resonance imaging. Ann N Y Acad Sci 649:204-224, 1992

11. Mislow JMK, Golby AJ, Black PM: Origins of intraoperative MRI. Neurosurg Clin N Am 20:137-146, 2009

12. Nuwer MR (ed): Intraoperative Monitoring of Neural Function. Handbook of Clinical Neurophysiology. Amsterdam: Elsevier, 2008

13. Schmitz BL, Aschoff AJ, Hoffmann MHK, Grön G: Advantages and pitfalls in 3T MR brain imaging: a pictorial review. AJNR Am J Neuroradiol 26:2229-2237, 2005

14. Shellock FG: Radiofrequency energy-induced heating during MR procedures: a review. J Magn Reson Imaging 12:3036,2000

15. Shellock FG, Woods TO, Crues JV III: MR labeling information for implants and devices: explanation of terminology. Radiology 253:26-30, 2009

16. Yarmolenko PS, Moon EJ, Landon C, Manzoor A, Hochman DW, Viglianti BL, et al: Thresholds for thermal damage to normal tissues: an update. Int J Hyperthermia 27:320-343, 2011

\section{Disclosures}

Dr. Roberts is a consultant with IMRIS Corp.

\section{Author Contributions}

Conception and design: Bauer, Darcey, Kobylarz, Roberts. Acquisition of data: Bauer, Darcey, Krauss, Pearl, Roberts. Analysis and interpretation of data: Darcey. Drafting the article: Darcey. Critically revising the article: Bauer, Kobylarz, Pearl, Ferri, Roberts. Reviewed submitted version of manuscript: Bauer, Darcey, Krauss, Pearl, Roberts. Administrative/technical/material support: Pearl, Ferri. Study supervision: Darcey.

\section{Correspondence}

David F. Bauer, Section of Neurosurgery, Dartmouth-Hitchcock Medical Center, 1 Medical Center Dr., Lebanon, NH 03756. email: david.f.bauer@hitchcock.org. 